\title{
Control of an Actuated Car Door Providing Outstanding Haptic Interaction
}

\author{
Michael Strolz* \\ Institute of Automatic \\ Control Engineering, \\ Technische Universität \\ München, Germany
}

\author{
Alexander Mörtl ${ }^{\dagger}$ \\ Institute of Automatic \\ Control Engineering, \\ Technische Universität \\ München, Germany
}

\author{
Michael Gräf: \\ BMW Forschung und \\ Technik GmbH, \\ Munich, Germany
}

\author{
Martin Buss ${ }^{\S}$ \\ Institute of Automatic \\ Control Engineering, \\ Technische Universität \\ München, Germany
}

\begin{abstract}
Actuated car doors are a promising way to increase the convenience of access to cars. In this paper, an advanced door concept which can easily be integrated into conventional car doors is presented. Using a linear, non-backdrivable actuator and various sensors, both automatic and manual door operations can be realized. The principal part is the realization of a high-quality haptic interaction of the car door for the manual operation. We implemented and tested four different impedance control schemes, of which impedance control with actuator force feedback performed best. This control scheme was subsequently used to haptically render different supportive dynamics and effects. An experimental evaluation with 16 participants revealed a predominant approval of the haptic sensation of the actuated car door.
\end{abstract}

\section{INTRODUCTION}

Even though a broad variety of new door concepts is exhibited in automotive fairs every year, conventional car doors with only one sliding or rotating, unactuated degree-of-freedom (DOF) are predominant in the market. Unfortunately, these doors exhibit a considerable discomfort to the user in some situations, e.g. while parking in a small lot or on a steep incline. Due to the often static door detent, the user may have to fix the door manually during egress to prevent damage to the door and adjacent cars. For moderate inclinations, this specific problem has recently been overcome by employing a (purely mechanical) variable door detent [8]. Using an actuator instead of such a mechanical door detent, the comfort and the safety of operation of the door can be increased:

- The door can automatically be opened and closed.

- In combination with a sensor system for the detection of obstacles, a collision prevention can be realized.

- The dynamic properties of the car door can be synthesized, and even be individually adjusted for every user, resulting in an "optimized" feel of the door.

Going even further, [9] proposed the use of actuated car doors with more than one DOF, allowing superior comfort during ingress. Unfortunately, building such a car door for the mass market would raise serious issues: Stiffness, weight and wear issues in the mechanics, guaranteeing safety of operation in the control, and changes in the assembly process in the production domain.

This motivates the design of an actuated car door deviating as little as possible from a conventional, rotational car door produced for the mass market. There have been various approaches to it, which were mainly published as patents.

\footnotetext{
*e-mail:strolz@tum.de

†e-mail:moertl@tum.de

†e-mail:michael.graef@bmw.de

§e-mail:m.buss@ieee.org
}

In [5], a vehicle door system comprising a magneto-rheological actuator is proposed. It allows the adjustment of the damping, thereby enabling several functionalities like a variable detent, deceleration and a limitation of the door opening. While this actuator has the benefit of being inherently safe, unfortunately it is only semi-active and cannot be used for creating an accelerating torque. This prevents both versatile haptic feedback and automatic operation of the door.

Several control systems are described that use one or more force sensors at the inner and/or the outer door handle to achieve a forcecontrolled motion of the car door that feels just like it is manually moved $[11,2,4]$. This assumes that the user can operate the door solely by gripping one of the door handles, which is not the case in everyday situations where many people hold the door at its upper corner. In [3], a door is described that is moved by an actuator, dependent on several sensor signals like inclination of the car, the force between actuator and door and information about obstacles in the workspace of the door. A different approach to modify the dynamics of the door are impedance or admittance control schemes, where motion sensors are used to measure the acceleration of the car door, see e.g. [7]. While these approaches contain good ideas, they do not comprise a combination of automatic and manual handling of the door with effective collision prevention. Furthermore, neither a detailed description of the actual implementation nor an evaluation of the haptic quality of these systems is available.

We discuss the development, control and evaluation of a mechatronic car door system, focusing on the haptic interaction between the human and the car door. By means of low-cost, state-of-the-art actuators, sensors and control technology, a conventional car door is redesigned to enhance safety and comfort to the user. Based on impedance control with force feedback, a superior manual handling of the car door is achieved which is intuitive and convenient. The sensor and control concepts have been implemented and evaluated on an experimental vehicle using rapid prototyping hardware.

\section{Modeling of the Actuated Car Door}

\subsection{Hardware Setup}

The complete experimental setup of the actuated car door can be seen in Fig. 1. A close-to-production linear actuator is integrated in the hollow space of the door. It is attached to a stiff location $(B)$ near the middle of the door, resulting in a high overall stiffness. Thus, a good power-flow between actuator and door is achieved. Several low-cost, partly redundant sensors are applied to the door system:

- High-bandwidth force sensor (1 DOF, in series with the driving rod of the actuator)

- Translational acceleration sensor (1 DOF, near external door handle, resolution $\Delta \ddot{x}<0.001 g$ )

- Analog (high-precision potentiometer at door hinge) and digital (self-made incremental encoder at the motor shaft, 480 counts/rev) position sensors (resolution of each: $\Delta \varphi \approx 0.06^{\circ}$ ).

Due to its collocation with the actuator, we used the digital sensor to achieve a high-bandwidth motion control. 


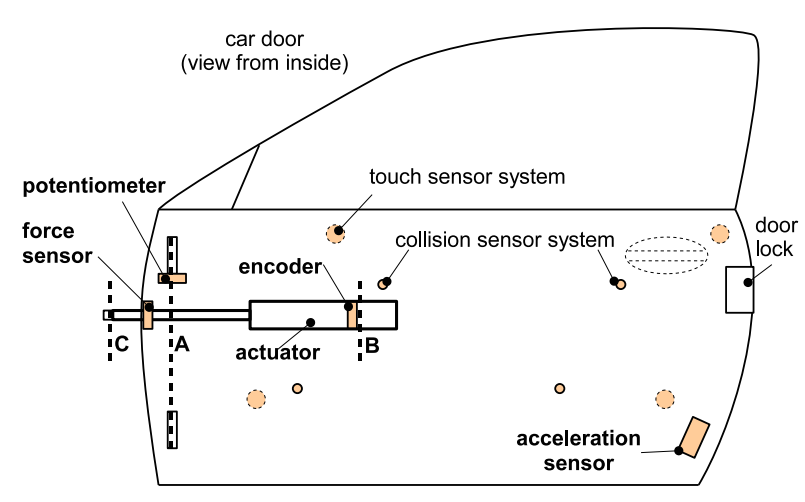

Figure 1: Hardware scheme of the actuated car door

Furthermore, several peripheral sensors are included in the test rig. The inclination of the car is measured by a two axis acceleration sensor, which is oriented in the horizontal plane. A new mechatronic door lock supports automatic opening and closing of the door. Proprietary systems for obstacle detection in the workspace of the door and detection of door touch by user, both based on ultrasonic transducers, are included in the test rig for demonstration purposes. The door lock, the sensors and the automatic control mode that they enable are not discussed in this paper. The control system is developed using MATLAB/Simulink and executed in real-time on a dSPACE AutoBox equipped with appropriate interface cards.

\subsection{Mechanical Modeling}

Due to the high stiffness of the door and the rigid structure of the actuation, the door system is idealized to be stiff. This leads to a simple kinematic model which is given in Fig. 2.

For the control design, the mapping between joint space and workspace (forward/inverse kinematics) has to be known. The angle of the door $\varphi$ is defined as workspace coordinate, while $s$ denotes the coordinate of the actuator. With the door hinge $\mathrm{A}$, the actuator bearing shafts B (at door) and C (at front column), and the geometrical parameters $l_{a}, l_{c}$ and $l_{m}$, the forward kinematics is given by

$$
\varphi=f(s)=\arccos \left(\frac{l_{a}^{2}+l_{c}^{2}-\left(l_{m}+s\right)^{2}}{2 l_{a} l_{c}}\right)-\varphi_{0}
$$

and the inverse kinematics is given by

$$
s=f^{-1}(\varphi)=\sqrt{l_{a}^{2}+l_{c}^{2}-2 l_{a} l_{c} \cos \left(\varphi+\varphi_{0}\right)}-l_{m}
$$

where $\varphi_{0}=f\left(l_{a}, l_{c}, l_{m}\right)=$ const. Due to deliberate mechanical design, $\varphi$ and $s$ are rather linearly linked for $0 \leq \varphi \leq 1.28 \mathrm{rad}$ (and $0 \leq s \leq 0.09 m$, respectively), which can also be seen from the Taylor series expansion of (2). This in turn provides a nearly linear mapping from the actuator force $F_{a}$ to the workspace torque $\tau_{a}$, which is important to avoid excessive actuator requirements. The mapping is described by the inverse Jacobian

$$
J(s)^{-1}=\frac{F_{a}}{\tau_{a}}=\frac{d \varphi}{d s}
$$

The dynamic behavior of the actuated car door can be described by its equations of motion:

$$
M(\varphi) \ddot{\varphi}+N(\varphi, \dot{\varphi})+G\left(\varphi, \gamma_{r}, \gamma_{p}\right)+J(s) F_{f}(\varphi, \dot{\varphi})=\tau_{a}-\tau_{e x t}
$$

where $M$ is the inertia of the moving parts, $N$ the Coriolis and centrifugal forces and $G$ the gravitational forces. It should be noted

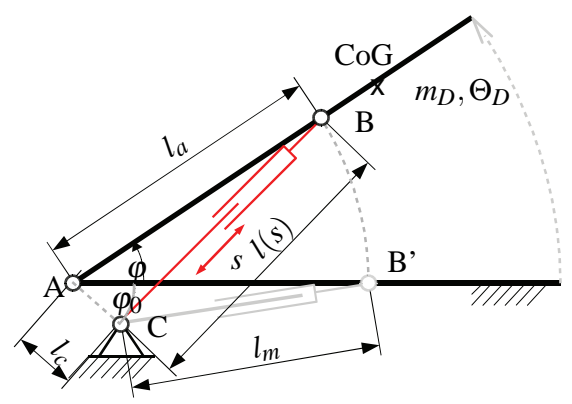

Figure 2: Kinematics of the door with prismatic actuator (top view)

that $G$ is determined by the inclination of both the car and the door hinge, given by $\gamma_{r}$ (roll) and $\gamma_{p}$ (pitch). Forces resulting from friction are split into

$$
F_{f}(\varphi, \dot{\varphi})=F_{f 1}+F_{f 2}
$$

where $F_{f 1}$ is the friction of the door hinge. Measurements revealed that this friction can accurately be modeled by a Coulomb friction. $F_{f 2}$ is a result of the friction of the actuator which is taken into account implicitly in Sec. 2.3. $\tau_{\text {ext }}$ is an external torque induced e.g. by a user and acts besides the actuation torque $\tau_{a}$.

\subsection{Modeling of the actuation}

The actuator consists of a brushed DC motor and a transmission, which is a combination of a planetary drive and a spindle (overall transmission ratio $r$ ).

Using standard, low-cost equipment, a high-bandwidth current control scheme can be implemented. For this reason, we assume both an ideal current control $\left(I=I_{r}\right)$ and a constant ratio $c_{m}$ of motor current $I$ and motor torque $\tau_{m}$, which gives $\tau_{m}=c_{m} I$.

To derive the transfer function of the actuator, we performed an experimental identification: The motor was controlled to a constant speed $n_{m}$. While measuring $n_{m}$ and $I$, we applied different constant forces $F_{a}$ on the linear rod. The identification revealed that $I$ can be modeled as a combination of two terms, one proportional to $F_{a}$ and the other nonlinearly depending on the velocity $\dot{s}$ :

$$
I=f\left(F_{a}, \dot{s}\right)=\frac{1}{c_{m} \eta\left(\tau_{m}, n_{m}\right) r} F_{a}+I_{0}(\dot{s})
$$

where $\eta\left(\tau_{m}, n_{m}\right)$ denotes the degree of efficiency of the transmission and $I_{0}(\dot{s})$ is the armature current without external actuator load $\left(F_{a}=0\right) . I_{0}(\dot{s})$ is proportional to the friction of the actuator $F_{f 2}$, which contains both a Coulomb and a viscous component, as can be seen in Fig. 3.

Based on this identification, we built a look-up table that is displayed in Fig. 4. Depending on the desired force $F_{a}$ and the motor speed $n_{m}$, the corresponding motor current $I$ is chosen by linear interpolation. To avoid discontinuities, a finite slope was chosen for the transition from small negative to small positive values of $n_{m}$.

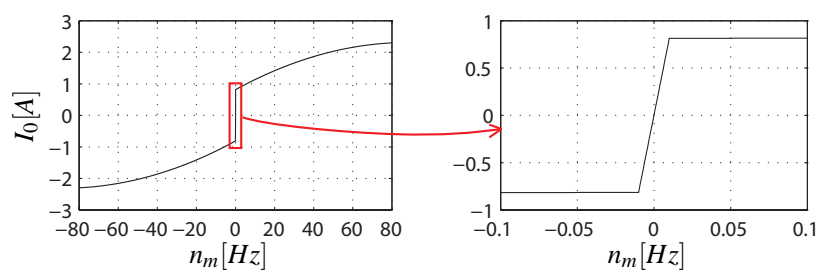

Figure 3: Speed-dependent friction component $I_{0}$ of motor current $I$ (l: full-scale view, $r$ : zoom that clarifies Coulomb friction influence) 


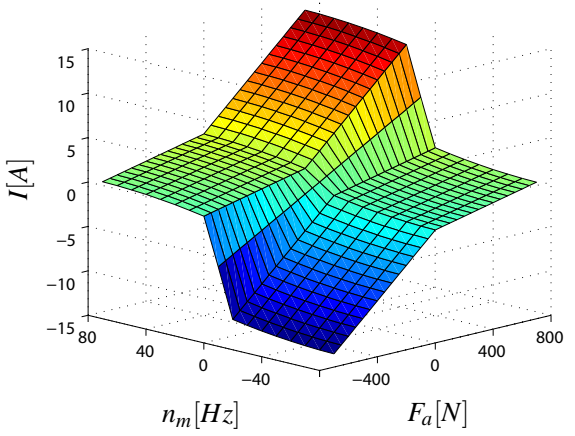

Figure 4: Look-up table for the calculation of motor torque $\tau_{m}=c_{m} I$ based on the motor speed $n_{m}$ and the desired actuator force $F_{a}$

\section{Manual Door Operation: Prearrangements}

Based on the models of the mechanics and the actuation, a controller for the manual operation of the car door can be implemented. Before doing so, we explain why we focused on impedance control schemes. Besides, we present two common components of them.

\subsection{Selection of impedance control}

By the use of kinesthetic feedback technology, we want to achieve a superior haptic interaction of the car door: Defined dynamic properties should be displayed with high quality. This includes defining the relation between the force $F$ and motion $\dot{x}$ of a rigid body, which can be done either by an impedance $Z=\frac{F}{\dot{x}}$ or an admittance $Y=\frac{\dot{x}}{F}$. Accordingly, such "virtual dynamics" are usually rendered by an impedance or an admittance controlled haptic device. A detailed overview of haptic control schemes is given in [12].

Impedance control does not require an explicit measurement of the interaction torque $\tau_{\text {ext }}$. This is a great benefit, because the reliable measurement of the interaction force at a car door is complex and expensive [10].

For haptic rendering, all following control concepts in this paper contain consistently the same virtual door impedance and thus are denoted as impedance control. To achieve good impedance control, the dynamics of the car door is compensated in part, see Sec. 3.2. The desired impedance is formed by superposition of the individual functional contributions explained in Sec. 3.3.

\subsection{Model feedforward}

Due to the lack of direct measurement of the user interaction force, the dynamic properties of the door cannot be shaped within a closed control loop when using impedance control. Therefore, based on (4) we do a feedforward compensation of the dynamics of the door:

$$
\tau_{f w d}=\tilde{M}(\varphi) \ddot{\varphi}+\tilde{N}(\varphi, \dot{\varphi})+\tilde{G}(\varphi)+\tilde{F}_{f 2}(\varphi, \dot{\varphi})
$$

While it was possible to fully compensate $N(\varphi, \dot{\varphi})$ and $G(\varphi, \dot{\varphi})$, $M(\varphi)$ could only be compensated in part $(\approx 40 \%)$ due to stability problems. It should be noted that this compensation requires an explicit measurement of $\ddot{\varphi}$. Furthermore, the friction of the door $F_{f 1}$ is not compensated to maintain stability, whereas $F_{f 2}$ is implicitly compensated by using the look-up table from Fig. 4.

Due to the compensation of $G(\varphi, \dot{\varphi})$, the inclination of the car does not affect the perceived dynamics of the car door. This provides a good comfort for the user when the car is inclined, because the user will not have to counteract gravity himself. Furthermore, it allows the use of even a large angle of inclination of the door hinge: The compensation spares the user the effort of counteracting the huge gravity force, and thereby makes such door kinematics acceptable.

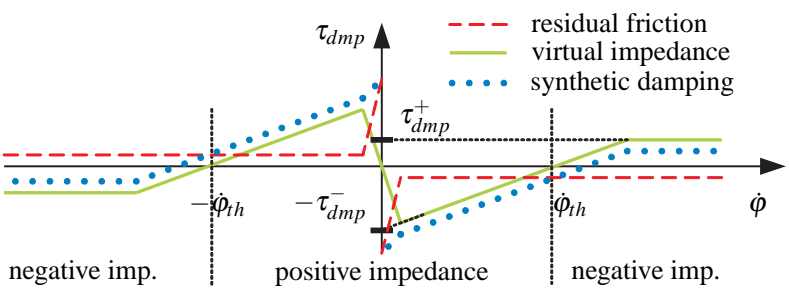

Figure 5: Synthetic damping (dotted), given by the residual friction (dashed) and the added virtual impedance (solid)

\subsection{Synthesis of the virtual door impedance}

Using an appropriate impedance, many different functionalities can be realized. In the following, three modules of the virtual door impedance are described which provide an active user assistance.

\subsubsection{Synthetic damping}

A variable damping is a key element in providing a situationdependent support: At standstill, an increased damping can help the user to overcome the breakaway torque (static friction) in a smooth manner. Furthermore, it could support the positioning of the door at low velocities. At higher velocities, when the user is thought to intend a full opening or closing of the door, a negative damping can support this motion.

To set up such a variable damping, a continuous virtual damping has to be defined that takes into account the residual friction of the door, i.e. the part of the physical friction that has not been compensated by the motion control (see Fig. 5, dashed line). We propose a virtual impedance with the damping characteristics

$$
\tau_{d m p}(\dot{\varphi})=\operatorname{sign}(\dot{\varphi}) \cdot \min \left[\frac{|\dot{\varphi}|-\dot{\varphi}_{t h}}{\dot{\varphi}_{t h}} \tau_{d m p}^{-}, \tau_{d m p}^{+}\right]
$$

where $\tau_{d m p}^{-}$and $\tau_{d m p}^{+}$are positive torque constants. This damping, which is shown in Fig. 5 (solid line), adds up with the residual friction to the overall synthetic damping ( Fig. 5, dotted line). The synthetic damping appropriately supports the user by impeding or supporting the motion of the door. Note that the velocity threshold $\dot{\varphi}_{t h}$ has to be tuned carefully and the transitions between the intervals of impedance should be continuous to achieve a good haptic feedback for the user.

\subsubsection{Variable door stop}

To provide a safe and smooth deceleration of the door based on a maximum opening angle $\varphi_{o b s}$ (possibly provided by a collision detection system), a variable door stop similar to [5] has been developed. It monitors the door in state space and applies an appropriate deceleration torque $\tau_{s t p}$ if $\varphi_{o b s}$ is likely to be violated. After that, a "stiff virtual wall" (PD controller) counteracts a violation of $\varphi_{o b s}$ for a certain time period. Finally, a pure D controller renders a viscous damping, which enables the user to move the (retarded) door even into the potential unsafe area. This might be necessary in case of an erroneous collision detection.

\subsubsection{Stepless door notch}

Furthermore, to prevent a drift of the door at standstill (due to wind, sensor noise, etc.), the physical static friction is enhanced by a stepless door notch functionality. It is built up with the active impedance

$$
\tau_{n c h}(\varphi, \dot{\varphi})=K_{p}\left(\varphi_{r}-\varphi\right)-K_{d} \dot{\varphi}
$$

which is hooked up in the actual position $\varphi_{r}$ of the door on engage, i.e. at $\dot{\varphi}_{r}=\dot{\varphi}=0 . K_{p}$ and $K_{d}$ are control gains subject to the state of the door, and release of the door notch can be done smoothly according to a displacement $|\Delta \varphi|$. 


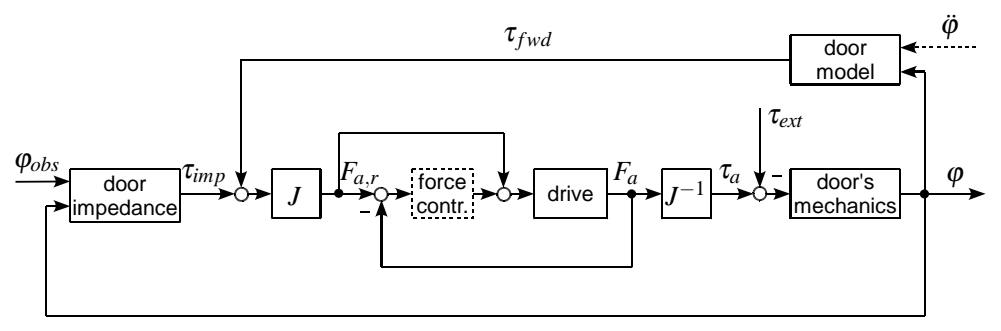

Figure 6: Impedance control of the car door; dotted block indicates actuator force feedback

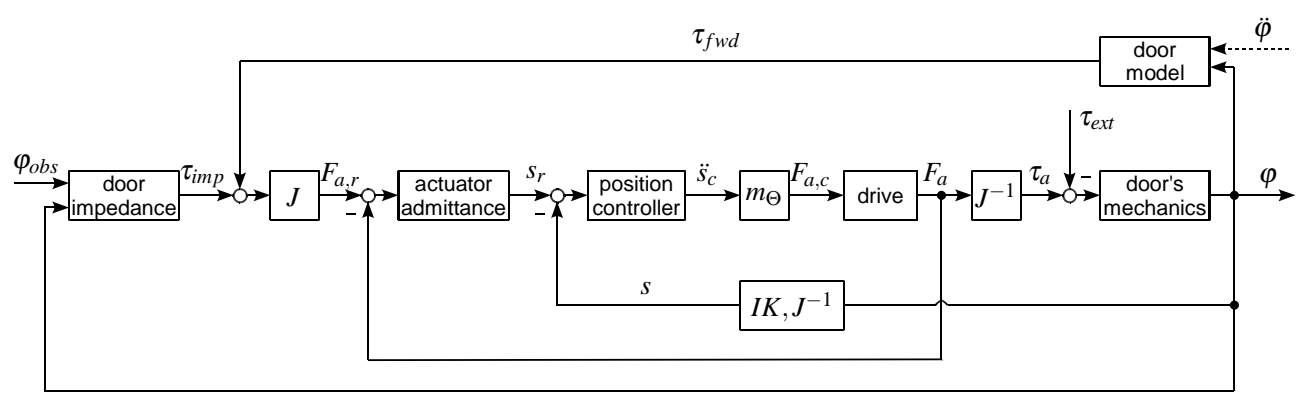

Figure 7: Position-based impedance control with force feedback

\section{Manual Door Operation: Control Schemes}

To achieve a good haptic rendering of the defined door dynamics, different impedance control schemes had to be implemented and compared to find the most suitable control concept. We found four promising, well-established impedance control concepts and implemented them heuristically such that they were stable for all relevant user interactions. In a subjective manner, their performance and the according hardware effort was evaluated experimentally.

\subsection{Impedance control without force feedback}

A simple implementation of impedance control is to employ openloop control of the actuator force:

$$
F_{a, r}=J\left(\tau_{i m p}+\tau_{f w d}\right)
$$

where $\tau_{i m p}$ and $\tau_{f w d}$ are the torques resulting from the virtual impedance and the door model respectively. Only force feedforward is active here, so the dotted block in Fig. 6 representing the force controller is set to zero.

One advantage of this concept is that it only requires the measurements of state $(\varphi, \dot{\varphi})$ and inclination $\left(\gamma_{r}, \gamma_{p}\right)$. It provided a quite good haptic sensation. However, it does not enable the compensation of the inertia of the door.

\subsection{Impedance control with force feedback}

A measurement of the actuator force $F_{a}$ allows the extension of the previous approach by explicit force control, see Fig. 6. Using a PD force-feedforward controller according to

$$
F_{a, c}=K_{p}\left(F_{a, r}-F_{a}\right)+K_{d} \frac{d}{d t}\left(F_{a, r}-F_{a}\right)+F_{a, r}
$$

where $K_{p}, K_{d}$ are controller gains. This control scheme provided a really good (subjective) feel of the door. Compared to other approaches, major advantages of this feedback of actuator force are 1) improvement of steady state accuracy of rendered forces in the presence of model uncertainties 2) good starting characteristics of the actuator due to effective reduction of static friction.

\subsection{Position-based impedance control with force feed- back}

A PD-type motion controller with acceleration feedforward and feedback linearization [12] is used for the control of the actuator state $(s, \dot{s})$, and a force $F_{a, c}=m_{\Theta} \ddot{s}_{c}$ is commanded with $m_{\Theta}$ being the inertia of the actuator. As can be seen in Fig. 7, this loop is driven by a model of the actuator, which gives the force control law

$$
F_{a, r}-F_{a}=m_{\Theta}^{\prime} \ddot{s}_{r}+d_{\Theta}^{\prime} \dot{s}_{r}
$$

It thereby requires the measurement of the actuator force $F_{a} . m_{\Theta}^{\prime}$ and $d_{\Theta}^{\prime}$ are the parameters of this admittance, which can be chosen by the system designer. They directly affect the overall dynamic perceived by the user. The benefits are 1) an improved rejection of unmodeled friction effects by use of a high gain motion control and 2) the possibility to virtually decrease the inertia of the door without a measurement of $\ddot{\varphi}$.

Unfortunately, due to the inherent gear backlash of the drive, this control concept yielded a rather limited performance.

\subsection{Position-based impedance control with force ob- server}

A combination of the virtual impedance defined in Sec. 3.3 and an admittance model of the door is proposed. This defines the overall system dynamics, as can be seen in Fig. 8 .

Again, a motion controller similar to [12] has been used, leaving out only the acceleration feedforward. Similar to (12) an admittance

$$
\tau_{\text {imp }}-\tau_{\text {ext }}=\Theta_{D}^{\prime} \ddot{\varphi}_{r}+d_{D}^{\prime} \dot{\varphi}_{r}
$$

is set up now, where $\Theta_{D}^{\prime}$ and $d_{D}^{\prime}$ are the desired inertia and damping of the actuated car door respectively.

The external interaction force $\tau_{\text {ext }}$ needed to drive the admittance (13) can be estimated by an observer $[6,1]$. Therefore, a model of the inverse dynamics of the door is used (see (4)).

For correct estimation of interaction force $\left(\hat{\tau}_{e x t}=\tau_{\text {ext }}\right)$ we assume that $\tau_{a, c}=\tau_{a}$ holds, so no explicit measurement of the actuator force is conducted. However, the observer requires the measurement of the acceleration $\ddot{\varphi}$. Advantages of this observer-based approach are 1) that the global inertia and damping of the door can 


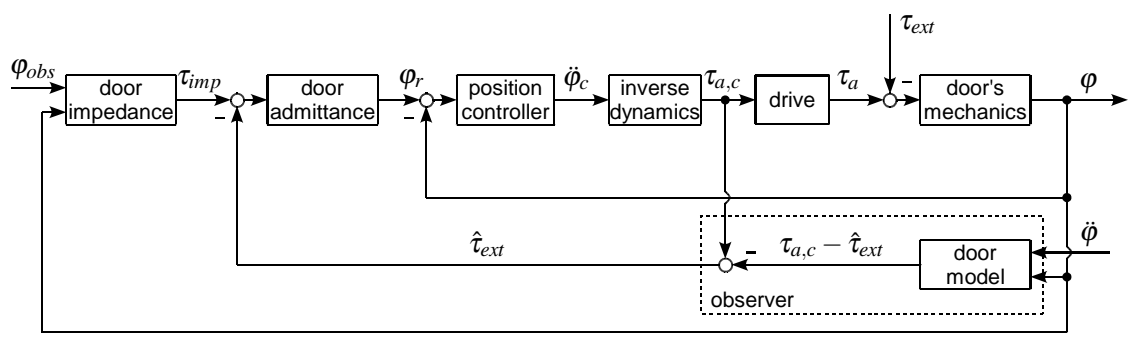

Figure 8: Position-based impedance control with force observer

be modeled with respect to the workspace coordinate $\varphi$ and 2) that nonlinearities (e.g. friction of door and actuator) are suppressed effectively.

However, the performance was only fair. The reason for this is that the actuation and the measurement of acceleration are not collocated. The finite structural stiffness of the mechanical elements in between accounts for higher-order dynamics, which have been neglected in the observer model.

\subsection{Conclusion}

The best (subjectively measured) performance of all four implementations was achieved by the impedance control with force feedback (Sec. 4.2). It requires the measurement of position, acceleration and actuator force, and thus is rather costly when compared to the three alternative control concepts. Nonetheless, as we believed that it yields the best haptic sensation, we chose it as reference setting for the evaluation described in Sec. 5.

From a performance point of view, the most promising alternative would be the use of impedance control without force feedback. Thus, the force sensor could be omitted, significantly lowering the cost of the overall system.

\section{Manual Door Operation: Evaluation}

We experimentally evaluated the manual door operation by a user study with 16 participants. The results indicate that the haptic interaction with this actuated car door is indeed outstanding.

\subsection{Design of the user study}

\subsubsection{Selection of the evaluation parameters}

The most relevant parameters for the haptic perception of the actuated car door were thought to be rendered mass, damping, deceleration and the parameters of the variable door stop (conventional door: $M \approx 26 \mathrm{~kg}, F_{f 1} \approx 3 \mathrm{Nm}$ ). The graduations described in Table 1 were thought to allow a quantitative assessment of the influence of these parameters on the haptic perception of the door. They contain a combination which provided a really good haptic interaction during development of the control concept: "natural" mass (m2), "low" damping (d2), stepless door notch "active", "high" deceleration, "short" release time and "high" stop damping. This combination was used as reference setting in the user study.

\subsubsection{Design of an evaluation sheet}

Based on Table 1, an evaluation sheet has been designed.

The first part consisted of four questions, which should give the general impression of the users: General usability (Q1), manual operation of the door $(\mathrm{Q} 2)$, equivalence of desired and actual motion (Q3) and the behavior at door stop (Q4). Possible answers were good (3), rather good (2), rather bad (1), and bad (0)).

The second part was designed to allow a full-factorial analysis of the influence of mass and damping on the haptic rendering of the door. This is done by allowing the participant to judge on each setting, e.g. " $\mathrm{m} 2 \mathrm{~d} 3$ ", in comparison to the reference setting " $\mathrm{m} 2 \mathrm{~d} 2$ ".
Possible answers were as follows: much better (2), better (1), no difference $(0)$, worse $(-1)$, and much worse $(-2)$.

The third part consisted of four questions, each going along with one variation of $\tau_{n c h}, \ddot{\varphi}, t_{r}$ and $K_{d}$ : Preferable without stepless door notch (Q5), with lower deceleration (Q6), with longer time for deactivating door stop (Q7), and with low damping (Q8)? These questions could be answered on a scale of 5 steps, analogous to the second part.

The evaluation finished with Q9, which equals Q1.

\subsection{Experiment and results}

We had 16 participants ( 15 male, 1 female). Their mean age was 42.4 years $(\sigma=11.06)$, and only one participant was not righthanded. All participants were employees of BMW. Thus, the group was surely not statistically matched to the general population of car door users. Indeed, we expected to get much more critical ratings on the performance of the actuated door, because many of these automotive experts are focused on achieving the best costumer acceptance for the individual car parts they design. Thereby, this group promised to give valuable hints on how the actuated car door performs and whether it would be accepted by potential customers.

The participants conducted the experiment in the order given by the evaluation sheet. We intentionally did not randomize the order of the questions in the first part, because Q1 and Q2 should be answered right before the user could significantly adapt to the novel door. This gives a valid estimate of the "first impression" of the door, which is considered to be an important criterion for the customer acceptance in the automotive industry. In the second part, we randomized the order of the mass-damping-settings to prevent learning effects. After this part, every participant had operated the door for more than 15 minutes. Therefore, we assumed that every user did get used to the door by then, such that no significant adaption would take place in part 3. Accordingly, Q5-Q8 were not randomized.

While the participants moved the door with the respective controller parameter setting, an investigator asked them the questions and filled out the the evaluation sheet. This might have slightly biased the evaluation of Q1-Q4 and Q9. However, we believe that this is not significant because of the professional participants. The results of the evaluation are displayed in Table 2.

Table 1: Evaluation Parameters for the User Study

\begin{tabular}{|c|c|c|c|}
\hline Parameters & \multicolumn{3}{|c|}{ Graduations } \\
\hline rel. mass $\frac{M^{\prime}}{M}$ & 'nat.', m2: 1 & 'high', m3: 1.4 & 'low', m1: 0.6 \\
\hline damp. $\frac{\tau_{d m p}^{+}}{\dot{\varphi}_{t h}}\left[\frac{N m s}{r a d}\right]$ & 'low', d2: 5 & 'high', d3: 8 & 'none', d1: 0 \\
\hline door notch $\tau_{n c h}$ & \multicolumn{2}{|c|}{ 'active" } & 'inactive" \\
\hline decel. $\ddot{\varphi}_{\max }\left[\frac{\mathrm{rad}}{\mathrm{s}^{2}}\right]$ & \multicolumn{2}{|c|}{ 'high': 1.75} & low': 1.00 \\
\hline release time $t_{r}[s]$ & \multicolumn{2}{|c|}{ 'short': 0.5} & long‘: 1.5 \\
\hline damping $K_{d}\left[\frac{N m s}{r d}\right]$ & \multicolumn{2}{|c|}{ 'high': 150} & 'low': 75 \\
\hline
\end{tabular}


Table 2: Evaluation results for the actuated car door based on a user study with 16 participants

\begin{tabular}{|c||c|c||c||c||c|c|}
\hline & mean & std.dev. & & & mean & std.dev. \\
\hline Q1 & 2.56 & 0.61 & & $\mathrm{~m} 1 \mathrm{~d} 1$ & -1.06 & 1.03 \\
Q2 & 2.44 & 0.70 & & $\mathrm{~m} 1 \mathrm{~d} 2$ & -0.31 & 1.21 \\
Q3 & 2.63 & 0.60 & & $\mathrm{~m} 1 \mathrm{~d} 3$ & -0.19 & 1.13 \\
Q4 & 2.38 & 0.78 & & $\mathrm{~m} 2 \mathrm{~d} 1$ & -0.31 & 0.68 \\
Q5 & -0.31 & 1.04 & & $\mathrm{~m} 2 \mathrm{~d} 2$ & - & - \\
Q6 & -0.88 & 1.11 & & $\mathrm{~m} 2 \mathrm{~d} 3$ & 0.06 & 1.03 \\
Q7 & -1.00 & 1.06 & & $\mathrm{~m} 3 \mathrm{~d} 1$ & -0.56 & 1.06 \\
Q8 & 0.13 & 1.17 & & $\mathrm{~m} 3 \mathrm{~d} 2$ & -0.38 & 0.99 \\
Q9 & 2.50 & 0.71 & & $\mathrm{~m} 3 \mathrm{~d} 3$ & -0.25 & 1.25 \\
\hline
\end{tabular}

\subsection{Analysis and discussion of the user study}

As can be seen from the mean of Q1-Q4, people liked the actuated car door and its features. Only few participants rated aspects to be "rather bad", and no one rated any aspect to be "bad". This suggests that the proposed concept would be accepted by customers.

The comparison of different mass and damping settings revealed that " $\mathrm{m} 2 \mathrm{~d} 2$ " and " $\mathrm{m} 2 \mathrm{~d} 3$ " were liked most. To analyze the results in detail, at first a two-factorial ANOVA (Analysis of Variance) was used. The $3 \times 3$ design that has been chosen allows the analysis of the influence of both factors (independent variables) on the manual operation of the car door (dependent variables). As threshold of significance $p=0.05$ was used. Under consideration of the sphericity, the mass showed to be not significant $(F(1.314,15)=1.019$, $\left.p>0.05, \eta^{2}=0.64\right)$. The damping proved to be significant $(F(2,15)=6.818, p<0.05)$. The interaction of both factors was not significant $(F(4,60)=0.815, p>0.05)$.

A pairwise comparison of all graduations of the damping according to the Bonferroni correction showed only for one pair a significant difference: "no damping" and "high damping" $(t(15)=0.521$, $p<0.05)$. Thus, regardless of the mass, a high damping seems to positively influence the haptic sensation of the car door in our setup.

The opinion about the stepless door notch was divided, see the results for Q5: One half of the participants liked this functionality, the other reported that it disturbed the operation of the door.

The mean values of Q6 and Q7 revealed that the deceleration should indeed be high and that the time for releasing the door right after a stop should be short, just as in the reference parametrization. However, according to Q8, there seems to be no clear tendency on the variation of the damping $K_{d}$.

A statistical evaluation revealed that two factors of the variable door stop were significant when comparing the reference setting with the alternative setting: The deceleration $(t(15)=8.05, p<$ $0.05)$ and the release time $(t(15)=3.651, p<0.05)$.

Interestingly, there were no significant differences in the general estimation of the actuated car door at the beginning and the end of the experiment: Q1 and Q9 lead to similar, very good results. From all 16 participants, a majority of 10 people rated the door with the best value of the given graduations, and another 4 people with the second best. This approval of $87.5 \%$ of the participants suggests that the actuated car door is not only liked right from the start by the users, but also after people get used to it.

\section{CONCLUSIONS}

In this paper, we showed a way to design an actuated car door such that potential customers like it both at first contact and after getting used to it. Furthermore, due to its clever mechanical concept, it can be built into conventional car doors without great modifications. Main components of the door are a linear drive, a current-controlled amplifier and sensors for position, acceleration and actuator force.

While this setup enables an advanced automatic door operation, our main focus here was the control and evaluation of the manual door operation. We implemented and tested four different impedance control schemes. Due to several reasons, the impedance control with force feedback was the best choice for this door. We used this control scheme to render specified impedances, i.e. haptic effects that are meant to support the user while he operates the door. One such effect was a variable door detent that allows to place the door at a desired location where it is fixed by a position controller.

A major advantage seemed to be the possibility to vary the dynamic properties of the door (mass, damping and "synthetic" haptic effects). Indeed, an evaluation with 16 participants revealed that some of the controller parameters had a statistically significant influence on the estimation of the users. For example, a higher damping was clearly preferred to a low damping.

The most important result of the evaluation was that a majority of $87.5 \%$ of the participants liked the car door, with $62.5 \%$ giving the highest grading. This approval was found both at the first contact of the participants with the novel door, and after they got used to it. From this we reason that the customer acceptance of our actuated car door would be relatively high and hence it would be promising to market such doors.

\section{ACKNOWLEDGEMENTS}

The authors would like to thank Raphaela Groten. Her help in setting up the user study and analyzing its results was very valuable. Furthermore, the authors would like to thank Nattika Tananimit. Her Master's thesis laid one of the foundations of this paper.

The research has been performed in the scope of the joint cooperation CAR@TUM between BMW Forschung und Technik GmbH and the Technische Universität München.

\section{REFERENCES}

[1] K. Eom, I. Suh, W. Chung, and S. Oh. Disturbance observer based force control of robot manipulator without force sensor. In Proceedings of the IEEE International Conference on Robotics and Automation (ICRA 1998), pages 3012-3017, Leuven, Belgium, May 1998.

[2] T. Ganz and J. Appel. Manuell betätigtes Antriebssystem für ein motorisch verstellbares Schließteil eines Fahrzeugs. DE19911592A1, 2000.

[3] J. Halbritter. Betätigungsvorrichtung für eine Fahrzeugtür. DE19927871A1, 1999.

[4] T. Kiyoshi and K. Mitsuhiro. Car door opening/closing device. JP09317323A, 1997.

[5] J. Maas and S. Kern. Mechatronic vehicle door assistant. In IEEE/ASME International Conference on Advanced Intelligent Mechatronics, pages 1-5, 2007.

[6] K. Ohishi, M. Miyazaki, and M. Fujita. Hybrid control of force and position without force sensor. In Proceedings of the IEEE International Conference on Industrial Electronics, Control and Instrumentation, pages 670-675, San Diego, CA, November 1992.

[7] K. Sasajima, K. Nagase, and T. Kuribayashi. Vehicle door operating apparatus. US005804937A, 1998.

[8] Stabilus. DORSTOP G2 Stepless Door Positioning System. Customer Satisfaction + Press Releases 2005/2006, May 2006.

[9] M. Strolz, Q. Mühlbauer, C. Scharfenberger, G. Färber, and M. Buss. Towards a generic control system for actuated car doors with arbitrary degrees of freedom. In Proceedings of IEEE Intelligent Vehicles Symposium (IV 2008), pages 391-397, Eindhoven, The Netherlands, June 2008.

[10] M. Strolz, G. Vasilev, and M. Buss. Sensor system for the determination of the interaction force at a vehicle door. In Sensoren und Messsysteme 2008, 14. Fachtagung Ludwigsburg, VDI-Berichte 2011, pages 811-820. VDI-Verlag Düsseldorf, 2008.

[11] N. Tomohiko and S. Hiroyuki. Opening and closing device of door for vehicle. JP05112128A, 1993.

[12] M. Ueberle and M. Buss. Control of kinesthetic haptic interfaces. In Proceedings of the IEEE/RSJ International Conference on Intelligent Robots and Systems (IROS 2004), Workshop on Touch and Haptics, Sendai, Japan, 2004. 